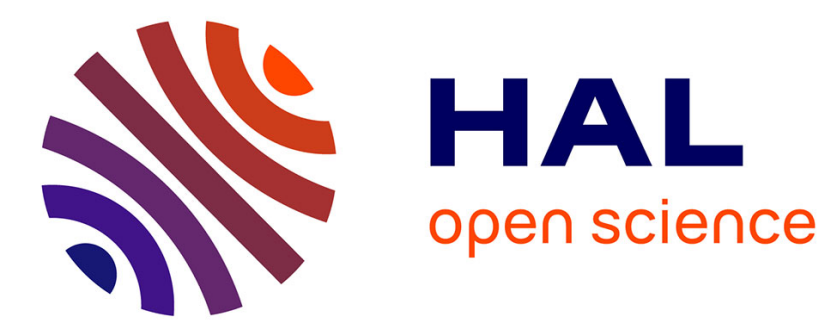

\title{
Deformation through Transformations
}

\author{
A. Roytburd
}

\section{To cite this version:}

A. Roytburd. Deformation through Transformations. Journal de Physique IV Proceedings, 1996, 06 (C1), pp.C1-11-C1-25. 10.1051/jp4:1996102 . jpa-00254133

\section{HAL Id: jpa-00254133 https://hal.science/jpa-00254133}

Submitted on 1 Jan 1996

HAL is a multi-disciplinary open access archive for the deposit and dissemination of scientific research documents, whether they are published or not. The documents may come from teaching and research institutions in France or abroad, or from public or private research centers.
L'archive ouverte pluridisciplinaire HAL, est destinée au dépôt et à la diffusion de documents scientifiques de niveau recherche, publiés ou non, émanant des établissements d'enseignement et de recherche français ou étrangers, des laboratoires publics ou privés. 


\title{
Deformation through Transformations
}

\author{
A.L. Roytburd \\ Material and Nuclear Engineering, University of Maryland, College Park, MD 20742, U.S.A.
}

\begin{abstract}
Constitutive stress-strain relations for transformational deformation are discussed. A crystal which can be in two possible phase states is considered. A phase transformation begins during the deformation after a certain amount of elastic strain. For each fixed level of strain an equilibrium polydomain microstructure is established which corresponds to a minimum in the free energy of the crystal. The equilibrium microstructure consists of plane-parallel layers of a product phase separated by layers of an initial phase. The product phase itself consists of different domains (twins) forming plane-parallel alternating layers. The volume fractions of the phases and the fractions of the different twin components in the product phase are functions of strain and temperature. The stress-strain curve which reflects the evolution of the equilibrium microstructure is calculated. For deformation under strain control the stressstrain curve has a section with negative or zero slope that corresponds to a negative or zero Young's modulus. If deformation proceeds under stress control, hysteretic stress-strain curves on loading and unloading will result from a section with negative slope.
\end{abstract}

\section{INTRODUCTION}

This paper is focused on deformation due to phase transformation. All phase transformations in solids are accompanied by some distortion or self-strain of a crystal lattice. This fact results in a special deformation mode of crystal during phase transformations. If the crystal remains coherent in a heterophase intermediate state, i.e. there is no plastic deformation or fracture, the transformational deformation can be structurally reversible. We will show here that it can be also thermodynamically reversible. Pseudoelastic, or superelastic (because the reversible deformation can be enormous) transformational deformation is the subject of this paper.

The mechanism of the transformational deformation is nucleation and movement of the interfaces, and the kinetics of the phase transformation determines the rate of the deformation. The analysis of the kinetics and interaction between phase transformation and dislocation deformation is very important for understanding pseudoelastic phenomena in real materials. However, these aspects of the transformational deformation will not be considered in this paper. We will show that a thermodynamics approach alone will lead to very interesting and unusual deformation behavior and our task is to describe it in detail.

In part two of this paper we consider the typical equilibrium microstructure during phase transformations and develop the concept of a polydomain phase. The strain controlled deformation through evolution of the equilibrium microstructure is analyzed in part three, the stress controlled - in part four.

\section{EQUILIBRIUM MICROSTRUCTURE AND $\sigma-\varepsilon$ RELATIONS FOR POLYDOMAIN PHASES}

Consider a crystal which can be in two possible phase states. The free energy of an uniformly distorted phase is determined by its strain but the two phases considered cannot be identical at the same strain. Ferroelastic, martensitic and order-disorder transformations of the first order are possible examples. The relative stability of the phases is determined by the difference of their free energies $\Delta f=\mathrm{f}^{0} 2^{-}$ $\mathrm{f}^{0}{ }_{1}$ where $\mathrm{f}^{0}{ }_{1}$ and $\mathrm{f}_{2}$ are free energies of undistorted initial and product phases, respectively. $\Delta \mathrm{f}$ is determined by temperature $T: \Delta f \approx\left(T-T_{0}\right) q / T_{0}$ where $q$ is a latent heat, and $T_{0}$ is an equilibrium 
temperature. Therefore, we will use $\Delta \mathrm{f}$ as a parameter which determined the temperature of isothermal transformational deformation.

In particular we consider the transformation of a cubic phase into a tetragonal one. In this case 3 different variants are formed with self- strains:

$$
\hat{\varepsilon}_{1}^{0}=\varepsilon_{0}\left(\begin{array}{ccc}
\chi & 0 & 0 \\
0 & -1 & 0 \\
0 & 0 & -1
\end{array}\right) ; \quad \hat{\varepsilon}_{2}^{0}=\varepsilon_{0}\left(\begin{array}{ccc}
-1 & 0 & 0 \\
0 & \chi & 0 \\
0 & 0 & -1
\end{array}\right) ; \quad \hat{\varepsilon}_{3}^{0}=\varepsilon_{0}\left(\begin{array}{ccc}
-1 & 0 & 0 \\
0 & -1 & 0 \\
0 & 0 & \chi
\end{array}\right)
$$

For $\chi=2$ these self-strains describe the transformation of the BCC to the FCC lattice ("Bain-strain"). This case, which corresponds to some martensitic transformations in $\mathrm{Cu}$-base shape memory alloys, is considered as an example.

The first problem which has to be considered is to find an equilibrium microstructure (fractions, morphology and arrangement of the phases) which has been established in a volume of the initial crystal if it is uniformly distorted and its surface is fixed. For solution of this problem we employ the equation of local equilibrium of interfaces (see e.g.[1]). This equation can be obtained by minimizing the free energy of two phases:

$$
F=\int_{V_{1}} f_{1}\left(\hat{\varepsilon}_{1}\right) d V+\int_{V_{2}} f_{2}\left(\hat{\varepsilon}_{2}\right) d V
$$

where $f_{1}$ and $f_{2}$ are the local free energies dependent on strains, $V_{1}$ and $V_{2}$ are the volumes of the phases, separated by the interface boundaries. An external surface of the two-phase crystal is fixed. The coherency of the phases means that the strains on the both side of the interface are compatible:

$$
\mathbf{n} \times\left(\hat{\varepsilon}_{1}-\hat{\varepsilon}_{2}\right) \times \mathbf{n}=0
$$

as well as in each points of the phase volumes

$$
\nabla \times \hat{\varepsilon} \times \nabla=0
$$

The equilibrium corresponds to vanishing of the variation of the functional (Eq.(2.2)) with respect to the strain variations and to displacement of the interface. Together with the compatibility of strains (Eq.(2.3) and Eq.(2.4)) it leads to the equations of the local equilibrium in the volume of the phases

$$
\nabla \cdot \hat{\sigma}=0
$$

where $\hat{\sigma}=\partial f / \partial \hat{\varepsilon}$ is a stress, and on the interface

$$
\left(\hat{\sigma}_{2}-\hat{\sigma}_{1}\right) \cdot \mathbf{n}=0
$$

and

$$
f_{2}-f_{1}-1 / 2\left(\hat{\sigma}_{1}+\hat{\sigma}_{2}\right)\left(\hat{\varepsilon}_{2}-\hat{\varepsilon}_{1}\right)=0
$$

Here are the stresses and the strains on the both sides of the interface at the point with normal $\mathbf{n}$.

If the linear elasticity of the phases is assumed, i.e. stresses are linear functions of strains:

$$
\hat{\sigma}_{1}=\mathbf{C}_{1}\left(\hat{\varepsilon}-\hat{\varepsilon}_{1}^{0}\right) ; \quad \hat{\sigma}_{2}=\mathbf{C}_{2}\left(\hat{\varepsilon}-\hat{\varepsilon}_{2}^{0}\right)
$$

where $\mathbf{C}_{1}$ and $\mathbf{C}_{2}$ are the elastic moduli and $\hat{\varepsilon}_{1}^{0}$ and $\hat{\varepsilon}_{2}^{0}$ are the self-strains, the free energies of the uniform phases are quadratic functions of the elastic strains $\left(\left(\hat{\varepsilon}-\hat{\varepsilon}^{0}\right)\right)$ :

$$
\begin{aligned}
& f_{1}=f_{1}^{0}+1 / 2\left(\hat{\varepsilon}-\hat{\varepsilon}_{1}^{0}\right) \mathbf{C}_{1}\left(\hat{\varepsilon}-\hat{\varepsilon}_{1}^{0}\right) \\
& f_{2}=f_{2}^{0}+1 / 2\left(\hat{\varepsilon}-\hat{\varepsilon}_{2}^{0}\right) \mathbf{C}_{2}\left(\hat{\varepsilon}-\hat{\varepsilon}_{2}^{0}\right)
\end{aligned}
$$


Then Eq.(2.7) transforms into

$$
\Delta f-1 / 2 \hat{\sigma}_{1}\left(\mathbf{S}_{2}-\mathbf{S}_{1}\right) \hat{\sigma}_{2}-1 / 2\left(\hat{\sigma}_{1}+\hat{\sigma}_{2}\right)\left(\hat{\varepsilon}_{2}^{0}-\hat{\varepsilon}_{1}^{0}\right)=0
$$

where $\mathbf{S}_{1}$ and $\mathbf{S}_{2}$ are the elastic compliances of the phases 1 and 2 , respectively.

It has been shown that for an uniformly stressed or strained initial crystal the solution of the equilibrium equations is the periodical alternation of the plane-parallel layer of the phases [2-4]. Some typical equilibrium microstructures which should be formed during the cubic-tetragonal phase transformation are shown in Fig.1.

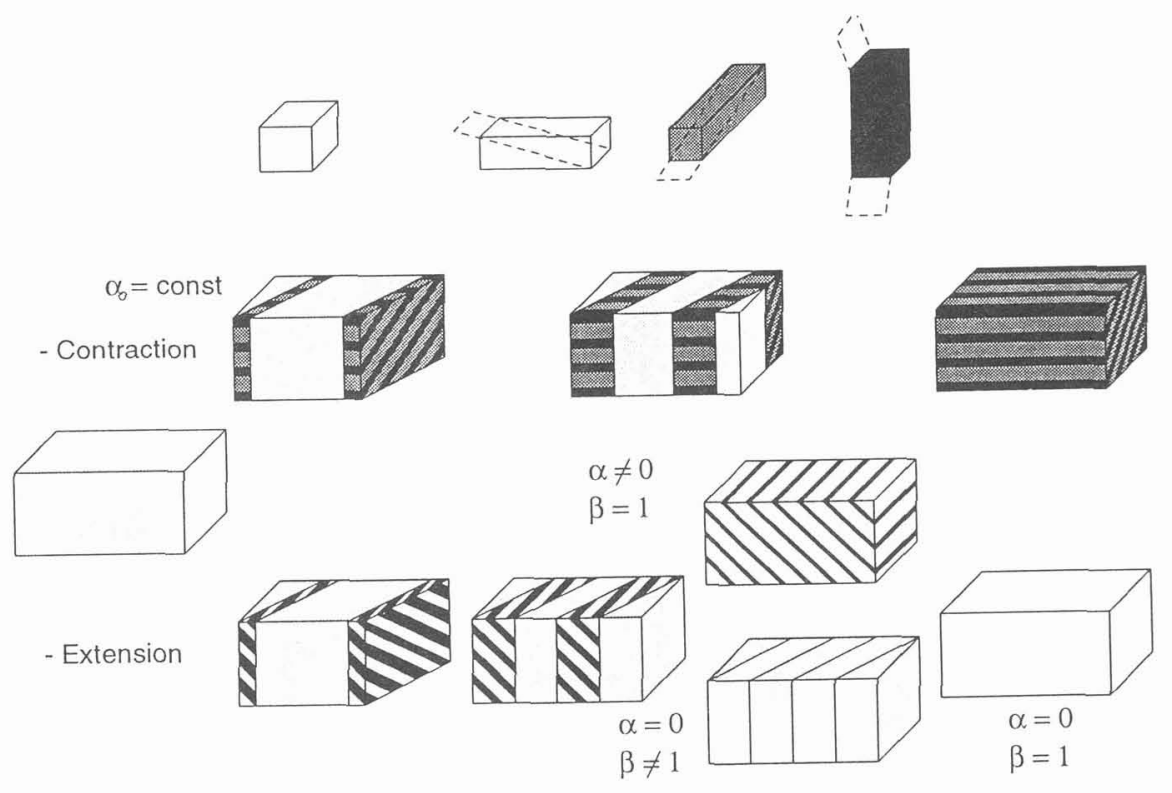

Figure 1: Evolution of microstuctures at uniaxial deformation

The simplest microstructures are formed by the layers of the initial phase and the single domain product phase (Fig.1a) and by the different domains of the product phases (Fig.1b). The orientation of interfaces in microstructure corresponds to minimum of the energy of internal stresses arising due to incompatibility of self-strains accross the interface. For the heterophase microstructure contained tetragonal domain 1, elastic adjustment of the phases is achieved as a result of an uniaxial extension along the axis [010] (which makes the strains along [010] equal in the both phases) together with a special orientation of the interface, that allows to obtain the compatibility without additional distortion. So the internal stress in this microstructure is uniaxial $[1,2,5]$.

In the case of domain layers the special orientation of the interfaces allows to get compatibility without any elastic distortion. The internal stresses are absent if domains border along the twinning plane (the plane $\{110\}$ for domains (2.1)). So, the equilibrium domain microstructures are the alternations of the plane parallel twins or a polytwin.. A polytwin can be considered as homogenous phase if a period of twin alternation is small in comparison with sizes of a reference crystal. In this case the contribution of the interfaces and the nonuniform distorsions near an external border is neglectable. The same is correct for a layer heterophase microstructure. This microstructure and a polytwin microstructure are examples of special polydomain phases. The twins as well as the phase layers accomodate external strain and decrease elastic energy, so they serve as an elastic domain $[1,2]$.

Beside the simplest microstructures considered above, microstructures of 2-nd order complexity are presented in Fig.1: layers of the product phase are polytwins. It gives an additional possibility to reduce incompatibility between the phases, because the self-strain of a polytwin is variable due to variation 
of domain fraction. On the other hand, including additional domains makes possible better adaptation of the external strain. The tendency to decrease internal elastic energy and to satisfy the external conditions can result in the formation of complex hierarchical microstructures (high order domain structures [2] or high rank laminate structures in modern terminology). However, complex microstructures can be less stable than more simple ones because of the increasing surface energy of interfaces. So, we restricted our consideration by the simple microstructures, given in Fig.1 which are at least metastable.

Because the phases in a layer microstructure are uniformly distorted, the free energy of the twophase system (Eq.2.2) can be rewritten as follows

$$
F=(1-\beta) f_{1}\left(\hat{\varepsilon}_{1}\right)+\beta f_{2}\left(\hat{\varepsilon}_{2}\right)
$$

where $\beta$ is a volume fraction of the phase 2 . The equilibrium fraction $\beta_{0}$ can be found by minimizing $F$ (Eq.(2.11)) $\mathrm{dF} / \mathrm{d} \beta=0$ with an additional condition:

$$
(1-\beta) \hat{\varepsilon}_{1}+\beta \hat{\varepsilon}_{2}=\overline{\hat{\varepsilon}}
$$

where $\bar{\varepsilon}$ is the fixed average strain given through the displacements on the surface. As result of strain compatibility

$$
\varepsilon_{1[010]}=\varepsilon_{2[010]}
$$

After $\hat{\varepsilon}_{1}\left(\beta_{0}\right)$ and $\hat{\varepsilon}_{2}\left(\beta_{0}\right)$ have been calculated the average stress can be obtained

$$
\overline{\hat{\sigma}}=\left(1-\beta_{0}\right) \hat{\sigma}_{1}+\beta \hat{\sigma}_{2}
$$

where $\hat{\sigma}_{1}$ and $\hat{\sigma}_{2}$ are related with $\hat{\varepsilon}_{1}$ and $\hat{\varepsilon}_{2}$ by Hook's law (Eq.(2.18)); $\beta_{0}$ is a function of $\overline{\hat{\varepsilon}}$. The relation between $\overline{\hat{\sigma}}$ and $\overline{\hat{\varepsilon}}$ gives the constitutive law for the transformational deformation. For polytwin product phase the self-strain is a function of the domain fraction $\alpha$. It gives an additional variable parameter for minimization of $F$.

If the difference between the elastic moduli of the phases is neglected the free energy (Eq.(2.11)) can be presented as follows:

$$
F=\Delta f \beta+1 / 2\left(\overline{\hat{\varepsilon}}-\beta \hat{\varepsilon}^{0}(\alpha)\right) \mathbf{C}\left(\overline{\hat{\varepsilon}}-\beta \hat{\varepsilon}^{0}(\alpha)\right)+1 / 2 \beta(1-\beta) E_{[010]} \varepsilon_{0}^{2}
$$

where $\mathbf{C}=\mathbf{C}_{1}=\mathbf{C}_{2}$ is the elastic modulus of both phases. Here the elastic energy is decomposed into two independent parts: the energy of an average ("external") strain $\left(\beta \hat{\varepsilon}^{0}(\alpha)\right.$ is an average self-strain) and the energy of the internal stresses ( $E_{[010]}$ is the Young's modulus in crystallographic direction [010]). This case of elastically identical phases will be considered hereafter. Elastic inhomogeneity brings in some additional effects, but makes the analysis much more complicated. Two additional simplifications will be used:

1. The phases are assumed to be elastically isotropic.

2. The external strain due to fixed boundary displacement results in the uniaxial stress.

These assumptions make the calculations simpler but does not change results in principle. The free energy for fixed extension or contraction along the axis $x_{1}, \bar{\varepsilon}$, is

$$
F=\Delta f \beta+1 / 2 E\left(\bar{\varepsilon}-\beta \varepsilon_{1}^{0}(\alpha)\right)^{2}+\beta(1-\beta) 1 / 2 E\left(\varepsilon_{3}^{0}(\alpha)\right)^{2}
$$

where $\varepsilon_{1}^{0}(\alpha)$ and $\varepsilon_{2}^{0}(\alpha)$ are principal strains of the polytwin product phase consisting of two types of domains. The physical meaning of this expression is illustrated by Fig.2. The second term represents the elastic energy of the uniform stress along $x_{1}$. The third one is the energy of internal stress which has only a component along $x_{3}$ with opposite directions in the different phases.

Minimizing $F$ 


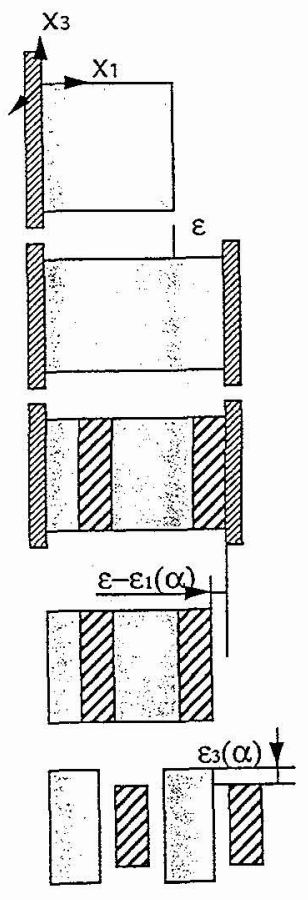

Figure 2: External and internal strains in a polydomain crystal

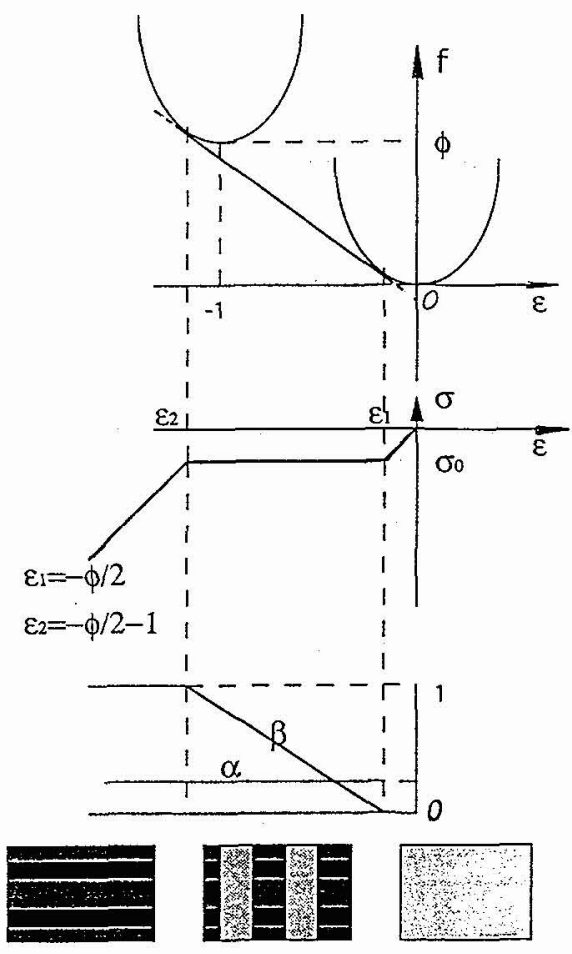

Figure 3: Equilibrium under contraction (free energy, stress-strain curve, microstructures)
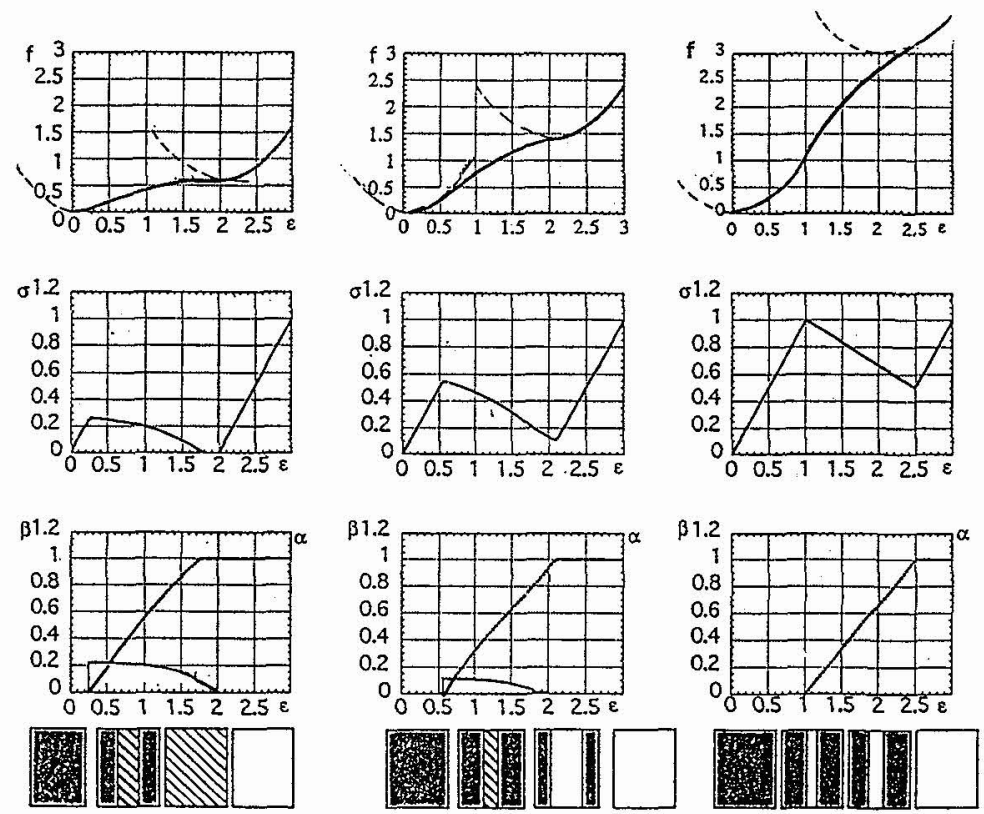

Figure 4: Equilibrium under extension $(\chi=2, \phi=0.6,2.2,3.4)$ 


$$
\frac{d F}{d \alpha}=0 \quad \frac{d F}{d \beta}=0
$$

the equilibrium $\alpha_{0}$ and $\beta_{0}$ as a function of $\bar{\varepsilon}$ are obtained and then the equibrium stress along $\mathrm{x}_{1}$ is calculated:

$$
\bar{\sigma}=E\left(\bar{\varepsilon}-\beta_{0} \varepsilon_{1}^{0}\left(\alpha_{0}\right)\right)
$$

where $\beta_{0} \varepsilon^{0}{ }_{1}(\alpha)$ is the self-strain along $x_{1}$.

\section{STRAIN CONTROLLED DEFORMATION}

Under contraction along axis $\mathrm{x}_{1}$ the equilibrium polytwin product phase consists of domains 2 and 3 with an average self-strain:

$$
\hat{\varepsilon}^{0}(\alpha)=(1-\alpha) \hat{\varepsilon}_{2}^{0}+\alpha \hat{\varepsilon}_{3}^{0}=\left(\begin{array}{ccc}
\varepsilon_{1}^{0}(\alpha) & 0 & 0 \\
0 & \varepsilon_{2}^{0}(\alpha) & 0 \\
0 & 0 & \varepsilon_{3}^{0}(\alpha)
\end{array}\right) \quad \begin{aligned}
& \varepsilon_{1}^{0}\left(\alpha^{\prime}\right)=-\varepsilon_{0} ; \\
& \varepsilon_{2}^{0}\left(\alpha^{\prime}\right)=\varepsilon_{0}(\chi-\alpha(\chi+1)) \\
& \varepsilon_{3}^{0}(\alpha)=\varepsilon_{0}(-1+\alpha(\chi+1))
\end{aligned}
$$

where $\alpha^{\prime}$ is a volume fraction of domain 3. In the equilibrium $\alpha^{\prime}=1 /(\chi+1), \varepsilon_{2}\left(\alpha^{\prime}\right)=0$ and internal stress is absent because the phase are compatible if they border along so called an invariant plane $[2,6]$. The free energy of the two-phases equilibrium state is

$$
f=\Delta f \beta+1 / 2 E\left(\varepsilon-\beta \varepsilon^{0}{ }_{1}\left(\alpha^{\prime}\right)\right)^{2}=\Delta f \beta+1 / 2 E\left(\varepsilon-\beta \varepsilon_{0}\right)^{2}
$$

because the therd term in $\operatorname{Eq}(2.16)$ is zero. The equilibrium fraction of the product phase is determined by equations $\partial \mathrm{f} / \partial \beta=0$ and equal to

$$
\beta_{0}=\Delta f / E \varepsilon_{0}^{2}-\varepsilon / \varepsilon_{0}
$$

It increases from 0 to 1 while $\varepsilon$ decreases from $\varepsilon_{1}=-\Delta f / E \varepsilon_{0}$ to $\varepsilon_{2}=-\Delta f / E \varepsilon_{0}-\varepsilon_{0}$. The stress remains constant $\sigma^{\prime}=-\Delta \mathrm{f} / \varepsilon_{0}$ during the transformation, i.e. the effective Young's modulus equals zero in the twophase state (Fig.3). The deformation of the coherent crystal is similar to compression of an equilibrium vapor-liquid mixture. The horizontal section of $\sigma-\varepsilon$ curve corresponds to the constant pressure section of the $\mathrm{P}-\mathrm{V}$ curve. The value of an equilibrium stress as a function of $\Delta \mathrm{f}$ or temperature gives the ClapeyronClausius relation which being inverted shows the stress dependence of the temperature of phase equilibrium.

The evolution of two-phase crystal during extension is considerably different from the evolution during contraction: (1) there is internal stress during deformation at any temperatures excepting the phase equilibrium temperature; (2) the microstructure of the product phase is variable and depends on the external strain [7].

The product phase contains the domain 1 which contributes to the maximum elongation along the extension axis. Therefore the polydomain phase should be a mixture of domain 1 and 2 (or 3 ). Thermodynamically domains 2 or 3 are equivalent and the polydomain consisting of domain 1 and 3 is considered (Fig.1). Its self-strain is

$$
\hat{\varepsilon}^{0}(\alpha)=(1-\alpha) \hat{\varepsilon}_{1}^{0}+\alpha \hat{\varepsilon}_{3}^{0}=\left(\begin{array}{ccc}
\varepsilon_{1}^{0}(\alpha) & 0 & 0 \\
0 & \varepsilon_{2}^{0}(\alpha) & 0 \\
0 & 0 & \varepsilon_{3}^{0}(\alpha)
\end{array}\right) \quad \begin{aligned}
& \varepsilon_{1}^{0}(\alpha)=\varepsilon_{0}[\chi-\alpha(\chi+1)] \\
& \varepsilon_{2}^{0}(\alpha)=-\varepsilon_{0} ; \\
& \varepsilon_{3}^{0}(\alpha)=\varepsilon_{0}[-1+\alpha(\chi+1)]
\end{aligned}
$$


where $\alpha$ is a fraction of domain 3 .

Thus the free energy of the two-phase state is:

$f=F / E \varepsilon_{0}^{2}=1 / 2 \phi \beta+1 / 2[\varepsilon-\beta(\chi-\alpha(\chi+1))]^{2}+1 / 2 \beta(1-\beta)[-1+\alpha(\chi+1)]^{2}$

where dimensionless parameters introduced are $\varepsilon=\bar{\varepsilon} / \varepsilon_{0} ; \phi=\Delta \mathrm{f} / 1 / 2 \mathrm{E}_{2} \varepsilon_{0}^{2}$. $\phi$ can be considered as the dimensionless temperature. The equations of equilibrium:

$$
\frac{\partial f}{\partial \alpha}=\frac{\partial f}{\partial \beta}=0
$$

determine the equilibrium microstructure, i.e. the equilibrium fractions of the product phase $(\beta 0)$ and of domain 3 in it $(\alpha 0)$ while the orientation of the interfaces between the phases is dependent on $\alpha_{0}$ and $\beta_{0}$. The solution of Eq.(3.6) is

$$
\alpha_{0}(1+\chi)=1-\varepsilon+\beta_{0}(\chi-1)
$$

with $\beta_{0}$ to be the solution of the equation

$\partial f / \partial \beta=1 / 2 \phi-1 / 2\left[\varepsilon-\beta_{0}(\chi-1)\right]^{2}-(1-\beta)[\varepsilon-\beta(\chi-1)](\chi-1)=0$

The equilibrium free energy is:

$$
f=1 / 2 \phi \beta_{0}+1 / 2(1-\beta)(\varepsilon-\beta(\chi-1))^{2}
$$

The dimensionless stress according to Eq. (3.7a) is :

$$
\sigma=\mathrm{d} f / \mathrm{d} \varepsilon=\bar{\sigma} / E \varepsilon_{0}=\varepsilon-\beta_{0}\left(\chi-\alpha_{0}(1+\chi)\right)=\varepsilon(1-\beta)+\beta(1-\beta)(\chi-1)
$$

An effective Young's modulus of the two-phase crystal is

$$
E_{\text {eff }}=\frac{d \sigma}{d \varepsilon}=\left(1-\beta_{0}\right)-\frac{\left[\varepsilon+\left(1-2 \beta_{0}\right)(\chi-1)\right]^{2}}{2\left[\varepsilon-\beta_{0}(\chi-1)\right](\chi-1)+\left(1-\beta_{0}\right)(\chi-1)^{2}}
$$

The strain dependencies of the equilibrium free energy, the stress and the modulus as well as the strain evolution of the microstructure for $\phi<1,1<\phi<2 \chi-1$ and $\phi>2 \chi-1$ are shown schematically in Fig.4. At $\phi>0$ the effective Young's modulus of the two-phase crystal is always negative and this fact corresponds to a convex free energy as a function of strain of the two-phase state.

There are four different sections of typical $\sigma-\varepsilon$ curves if $1<\phi<2 \chi-1$. These sections are separated by singular points $\left(\varepsilon_{1}, \sigma_{1}\right) ;\left(\varepsilon_{2}, \sigma_{2}\right)$ and $\left(\varepsilon_{3}, \sigma_{3}\right)$ where the microstructure dramatically changes with the external strain.

The section $0-\varepsilon_{1}$ corresponds to elastic deformation of the crystal: $\sigma=\varepsilon$. The first plates of polydomain product phase appear at strain $\varepsilon_{1}$. The value of $\varepsilon_{1}$ depends on $\phi$ and therefore, the equilibrium domain composition $\alpha_{0}$ (Eq.(3.7a)) depends on $\phi$ through its dependence on $\varepsilon_{1}$. Strain $\varepsilon_{1}$ and the equilibrium twin fraction $\alpha_{0}\left(\varepsilon_{1}\right)$ at the beginning of the transformation are determined by Eq.(3.7b) when $\beta_{0}=0$ :

$$
\varepsilon_{1}=-(\chi-1)+\left((\chi-1)^{2}+\phi\right)^{1 / 2}, \quad \sigma_{1}=\varepsilon_{1}
$$




$$
\alpha_{0}\left(\varepsilon_{1}\right)=\frac{1-\varepsilon_{1}}{\chi+1}=\frac{1}{\chi+1}+\frac{(\chi-1)-\left((\chi-1)^{2}+\phi\right)^{1 / 2}}{\chi+1}
$$

At the temperature of the phase equilibrium (when $\phi=0) \varepsilon_{1}=0$ and $\alpha_{0}\left(\varepsilon_{1}\right)=\frac{1}{\chi+1}$. The plate with this domain fraction has no elastic energy (because the self-strain reduces to an invariant plane strain). When $\phi$ increases $\varepsilon_{1}$ grows and $\alpha_{0}\left(\varepsilon_{1}\right)$ decreases; $\alpha_{0}\left(\varepsilon_{1}\right)$ approaches zero at $\phi=2 \chi-1$. The Young's modulus according to $(3.10)$ is

$$
E_{e f f}\left(\varepsilon=\varepsilon_{1}\right)=1-\frac{\phi+(\chi-1)^{2}}{\phi+(\chi-1)^{2}-\left(\sqrt{\phi+(\chi-1)^{2}}-(\chi-1)\right)^{2}}
$$

It is negative and its absolute value grows from 0 at $\phi=0$ up to $-1 /\left(\chi^{2}-1\right)$ at $\phi=2 \chi-1$.

The domain fraction $\alpha_{0}$ also decreases under isothermal deformation when the strain $\varepsilon$ increases beyond $\varepsilon_{1} . \alpha_{0} \rightarrow 0$ when $\varepsilon$ obtains the value $\varepsilon_{2}$. This value and $E\left(\varepsilon_{2}\right)$ as well as the corresponding value of phase fraction $\beta_{0}$ can be found from Eqs.(3.7), if $\alpha_{0}=0$ :

$$
\begin{aligned}
& \beta_{0}=1-(1 / 2) \frac{\phi-1}{\chi-1} \\
& \varepsilon_{2}=1+\beta_{0}(\chi-1)=\chi-(1 / 2)(\phi-1) \\
& \sigma_{2}=\varepsilon_{2}-\beta_{0} \chi=1-\beta_{0}=(1 / 2) \frac{\phi-1}{\chi-1} \\
& E_{e f f}\left(\varepsilon=\varepsilon_{2}\right)=-\frac{2}{(\chi-1)(\phi+3)}
\end{aligned}
$$

At the point $\left(\varepsilon_{2}, \sigma_{2}\right)$ the polydomain product phase degenerates into a single domain. Then the free energy of the two-phase system is

$$
f=1 / 2 \phi \beta+1 / 2(\varepsilon-\beta \chi)^{2}+1 / 2 \beta(1-\beta)
$$

The stress-strain curve reduces to a linear relation:

$$
\sigma=\varepsilon-\beta_{0} \chi=-\frac{1}{\chi^{2}-1} \varepsilon+\frac{\chi(1+\phi)}{2\left(\chi^{2}-1\right)} \quad ; \quad \beta_{0}=\frac{2 \chi \varepsilon-1-\phi}{2\left(\chi^{2}-1\right)}
$$

The Young's modulus can then be obtained as

$$
\mathrm{E}=d \sigma / d \varepsilon=-1 /\left(\chi^{2}-1\right)
$$

The fraction $\beta_{0}$ of single domain product phase grows with $\varepsilon$ and the transformation is complete $\left(\beta_{0}=1\right)$ at

$$
\varepsilon_{3}=\left(\chi^{2}-1\right) / \chi+(\phi+1) / 2 \chi, \quad \sigma_{3}=\varepsilon_{3}-\chi=(\phi-1) / 2 \chi
$$

Beyond strain $\varepsilon_{3}$, the product phase deforms elastically: $\sigma=\varepsilon-\chi$ (i.e. $\bar{\sigma}=\mathrm{E}\left(\varepsilon-\chi \varepsilon_{0}\right)$ ). 


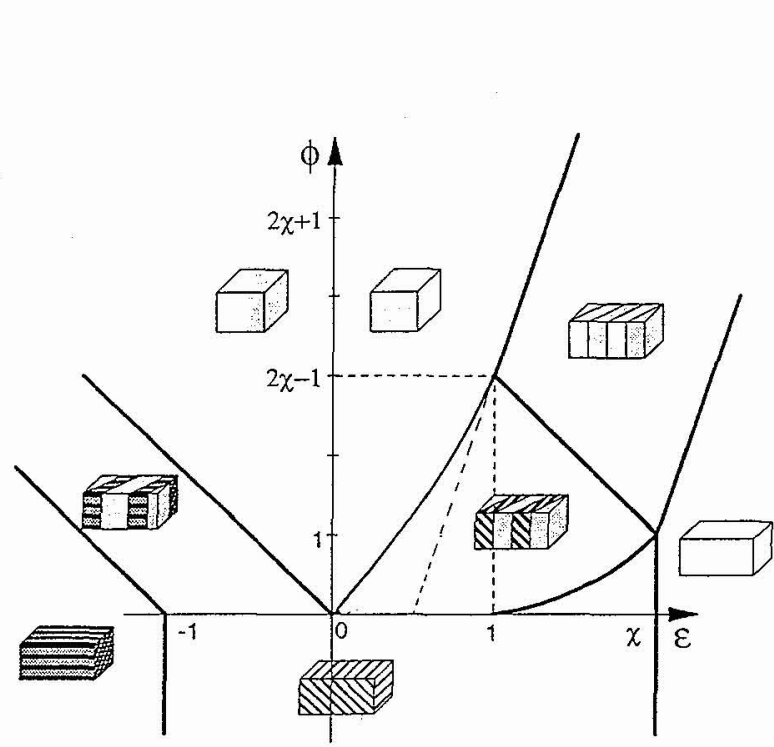

Figure 5: Strain-temperature diagram of equilibrium microstructures

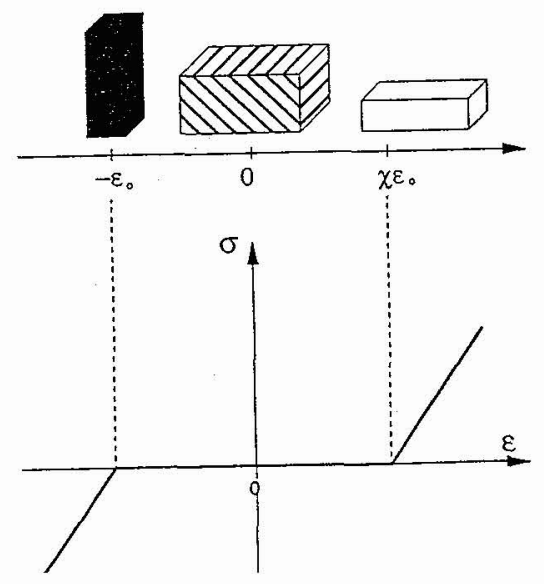

Figure 7: Strain controlled deformation of a polydomain crystal $(\phi<0)$

Figure 8: Displacement controlled deformation with negative Young's modulus

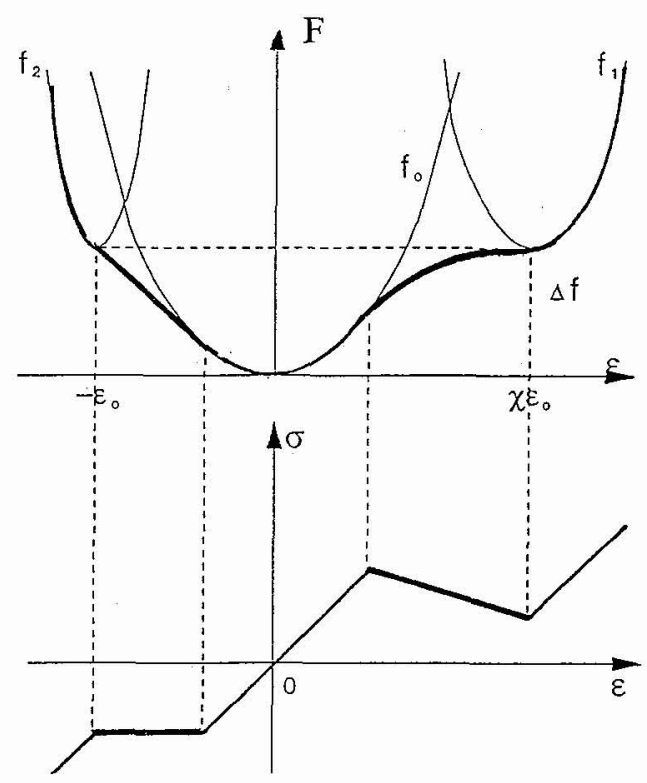

Figure 6: Strain-controlled deformation of a polydomain crystal $(\phi>1)$
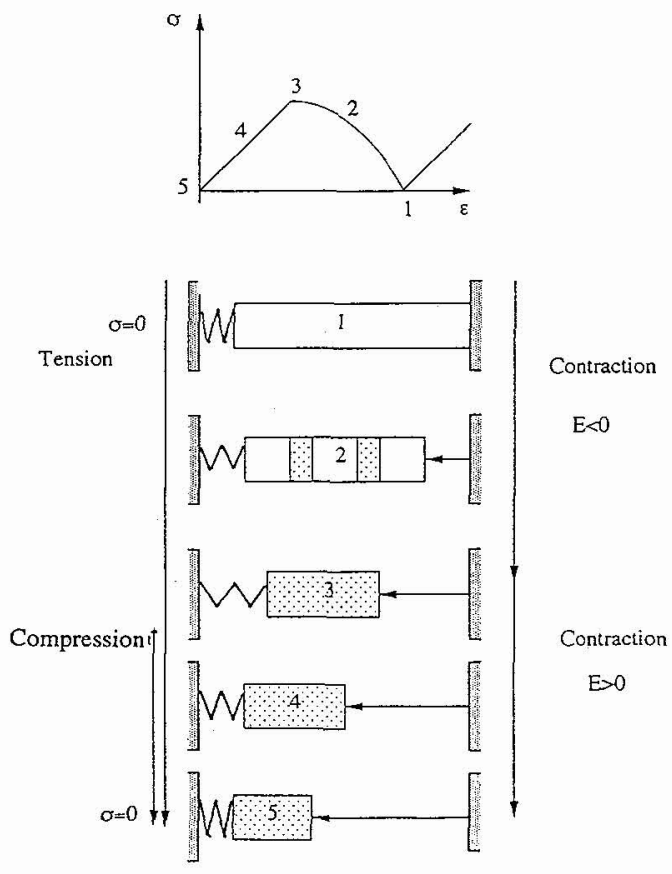
All these 4 stages of the coherent phase deformation exist only if $1<\phi<2 \chi-1$. At $\phi>2 \chi-1$ only the single domain phase arises and grows under strain. So $\varepsilon_{2}$ coincides with $\varepsilon_{1}$. The onset of the transformation with formation of the single domain product corresponds to the solution of Eqs.(3.7) at $\alpha_{0}=\beta_{0}=0$ :

$$
\varepsilon_{1}^{\prime}=(1+\phi) / 2 \chi, \quad \sigma_{1}^{\prime}=\varepsilon_{1}^{\prime}
$$

At $\phi<1 \quad \varepsilon_{3}$ is less than $\varepsilon_{2}$ : it means that the transformation is completed before the domain structure of the product phase disappears. From Eqs.(3.7) at $\beta_{0}=1\left(\alpha_{0}<1\right)$ it follows:

$$
\begin{aligned}
& \varepsilon_{3}^{\prime}=\chi-1+\sqrt{\phi} \quad \sigma_{3}^{\prime}=0 \\
& \alpha_{3}^{\prime}=\frac{1-\sqrt{\phi}}{\chi+1} \\
& E_{e f f}\left(\varepsilon=\varepsilon_{3}^{\prime}\right)=-\frac{1}{2(\chi-1)} \sqrt{\phi}
\end{aligned}
$$

Further deformation of the polydomain phase by detwinning can proceed at zero stress corresponding to the horizontal section on the two-phase free energy curve: $\operatorname{Eq}(3.5)$ transforms at $\phi=0, \beta=1$ into

$$
\mathrm{f}=\left(\varepsilon-\varepsilon_{1}(\alpha)\right)^{2}
$$

The free energy as well the stress and the modulus equal to zero if $\varepsilon_{1}(\alpha)=\varepsilon$ and $\alpha=(\varepsilon-\chi) /(\chi+1)$.

Thus the deformation of a two-phase coherent system with a polydomain product phase proceeds in a triangle in the $\sigma-\varepsilon$ plane with the apexes $(0,0),(1,1),(\chi, 0)$ and with the sides formed by the straight lines: $\sigma=\varepsilon_{1}$, the abscissa axis and the diagonal line which is the locus of points $\varepsilon_{2}, \sigma_{2}$ :

$$
\sigma_{2}=-\varepsilon_{2} /(\chi-1)+\chi /(\chi-1)
$$

Beyond this triangle a single domain product is formed. In the equilibrium two-phase mixture the tetragonal phase is in a single domain state at $\phi>2 \chi-1$ and in a polydomain state at $\phi<1$. At intermediate temperatures, the equilibrium state of the tetragonal phase changes with deformation: from a polydomain state to a single domain one.

The equations (3.3), (3.11), (3.13), (3.17), (3.18), (3.19) determine the area of stability of different microstructures on the plane $(\phi, \varepsilon)$ (Fig.5). At the temperatures lower than $\phi=0$ the free energy of the single phase product is lower than the energy of the two-phase state. The different constitutive laws corresponds to different areas of this diagrams. For example, the $\sigma-\varepsilon$ curves for temperatures higher than $2 \chi-1$ and lower than 0 are shown in Figs. 6 and 7.

Finally, we want to illustrate the concept of a negative modulus by a simple model in Fig.8. The spring indicates the stress during contraction of the sample from an initial tetragonal state. Due to the negative Young's modulus of the sample at the first stage of deformation, the spring indicates the tension stress on contraction of the sample. After the transformation is complete, the deformation of the sample in a cubic state proceeds with a normal positive Young's modulus and contraction results in compression. It is necessary to reiterate that only strain, or displacement controlled deformation is considered here. This means that the "free" surface of the sample is under the constraint, therefore the surface remains macroscopically smooth. However, there are no surface forces.

\section{STRESS CONTROLLED DEFORMATION}

The deformation through coherent phase transformation considered above corresponds to the evolution of an equilibrium heterophase mesostructure. The equilibrium is stable if the external strain is fixed. However, the equilibrium becomes unstable if the external stress is fixed instead of the strain. It results in a hysteretic $\sigma-\varepsilon$ relation for stress controlled deformation. 
The equilibrium under stress is determined by the minimum in Gibbs free energy and the work of the external stress $(-\overline{\hat{\sigma}} \hat{\varepsilon})$ should be added to the free energy $(\mathrm{Eq}(2.16))$.

$$
G=F-\overline{\hat{\sigma}} \cdot \overline{\hat{\varepsilon}}=\Delta f \beta-\bar{\sigma} \beta \varepsilon_{1}(\alpha)+\beta(1-\beta) 1 / 2 E\left(\varepsilon_{3}(\alpha)\right)^{2}-\sigma^{2} / 2 E
$$

Here it is taken into consideration, that $\bar{\sigma}=E\left[\bar{\varepsilon}-\beta \varepsilon_{1}(\alpha)\right]$

Equilibrium microstructures (determined by the equations $\mathrm{dG} / \mathrm{d} \alpha=\mathrm{dG} / \mathrm{d} \beta=0$ ) correspond to saddle points of the Gibbs free energy and they are unstable with respect to changing $\beta$.

Indeed, for compression $(\sigma<0)$ the dimensionless Gibbs free energy of the mixture of the cubic phase and the polytwins $(2,3)$ is

$$
g=G / E \varepsilon^{2} 0=\phi \beta / 2-\sigma^{2} / 2+\sigma \beta
$$

which corresponds to the minimum with respect to $\alpha$ (the coherent energy is disappears in Eq.(4.1) at $\alpha=1 /(1+\chi)$ ). This Gibbs free energy is a linear function of $\beta$ and obtains the minimum value $g_{0}=-1 / 2 \sigma^{2}$ at $\beta=0$ (the cubic phase) if $-\phi / 2<\sigma<0$. It takes the minimum value $\mathrm{g}_{23}=-\sigma^{2} / 2+\sigma+\phi / 2$ at $\beta=1$ (polytwin product $(2,3)$ ) if $\sigma<-\phi / 2$. The stress $\sigma_{0}^{\prime}=-\phi / 2$ determines the point of the equilibrium between two phases (Fig.9a). The definition of strain $\varepsilon=-\mathrm{dg} / \mathrm{d} \sigma$ leads to a strain-stress relation for stress controlled deformation:

$$
\sigma=\left\{\begin{array}{cc}
\varepsilon & -\phi / 2<\varepsilon<0 \\
-\phi / 2 & -(\phi / 2+1)<\varepsilon<-\phi / 2 \\
\varepsilon+1 & \varepsilon<-(\phi / 2+1)
\end{array}\right.
$$

This relation is identical to the $\sigma-\varepsilon$ relation for strain controlled deformation (Fig.3).

For tension the Gibbs free energy of a mixture of the cubic phase and the polytwin $(1,3)$ is:

$$
g(\alpha, \beta)=-1 / 2 \sigma^{2}+\phi \beta / 2-\sigma \beta[\chi-\alpha(\chi+1)]+1 / 2 \beta(1-\beta)\left[(-1+\alpha(\chi+1)]^{2}\right.
$$

It transforms into the Gibbs energies of the cubic phase, $g_{0}$, (at $\left.\beta=0\right)$, the tetragonal single domain phase, $\mathrm{g}_{1}$, (at $\beta=1, \alpha=0$ ) and the polytwin tetragonal phase, $\mathrm{g}_{13}$, (at $\beta=1, \alpha=1 /(\chi+1)$ ).

$$
g_{0}=-1 / 2 \sigma^{2} \quad g_{13}=-1 / 2 \sigma^{2}+1 / 2 \phi-\sigma(\chi-1) \quad g_{1}=-1 / 2 \sigma^{2}+1 / 2 \phi-\sigma \chi
$$

At $(\phi-1) /\left(2(\chi-1) \leq \sigma \leq \phi / 2(\chi-1) \mathrm{g}(\alpha, \beta)\right.$ has a saddle point $\left(\mathrm{dg} / \mathrm{d} \alpha=\mathrm{dg} / \mathrm{d} \beta=0 \quad \mathrm{~d}^{2} \mathrm{~g} / \mathrm{d} \alpha^{2}>0, \mathrm{~d}^{2} \mathrm{~g} / \mathrm{d} \beta^{2}<0\right)$

$\alpha^{*}=\frac{1}{\chi+1}(1-\sqrt{2 \gamma}) ; \quad \beta^{*}=1-\frac{\sigma}{\sqrt{2 \gamma}} \quad g^{*}=\gamma-\sigma \sqrt{2 \gamma}$

where $\gamma=\phi / 2-\sigma(\chi-1)=\mathrm{g}_{13}-\mathrm{g}_{0}$ is a driving force for transformation $0 \rightarrow 13$.

At $\sigma<\sigma_{2}=(\phi-1) / 2(\chi-1), \alpha^{*}=0$ and the saddle point degenerates into a maximum of $g(\alpha=0, \beta)$ :

$$
\beta^{*}=\delta+1 / 2 \quad g^{*}=-\delta^{2} / 2+1 / 2(\delta+1 / 2)^{2}
$$

where $\delta=\phi / 2-\sigma \chi=\mathrm{g}_{1}-\mathrm{g}_{0} . \delta=0$ corresponds to the equilibrium of the isolated phases 0 and 1 at the equilibrium stress $\sigma_{0}=\phi / 2 \chi$. 


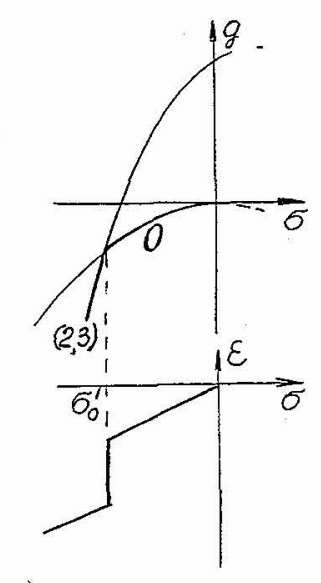

a)

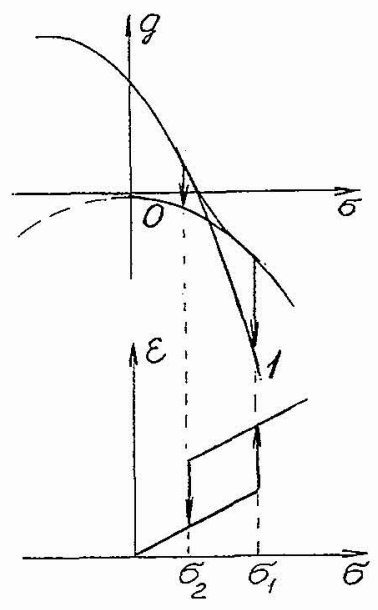

Figure 9: Equilibrium under stress. Gibbs free energies and stress-strain curves, a -compression, b- tension

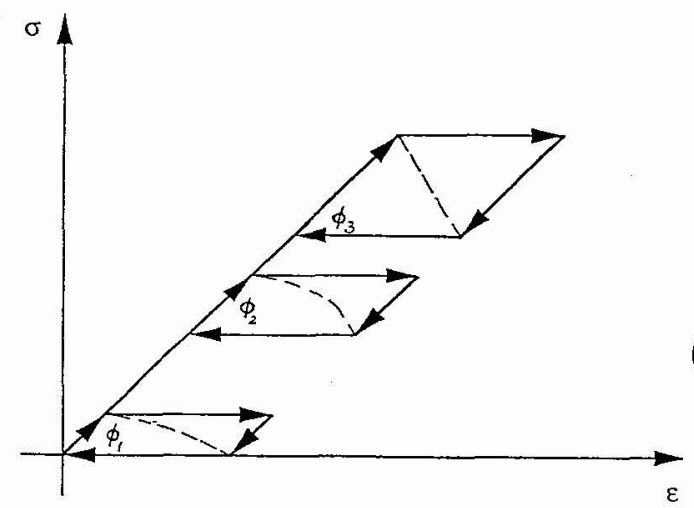

Figure 10: Stress controlled deformation, temperatures: $\phi_{1}<1,1<\phi_{2}<2 \chi-1$ $\phi_{3}>2 \chi-1$

Figure 11: Stress-temperature diagram of equilibrium microstructures

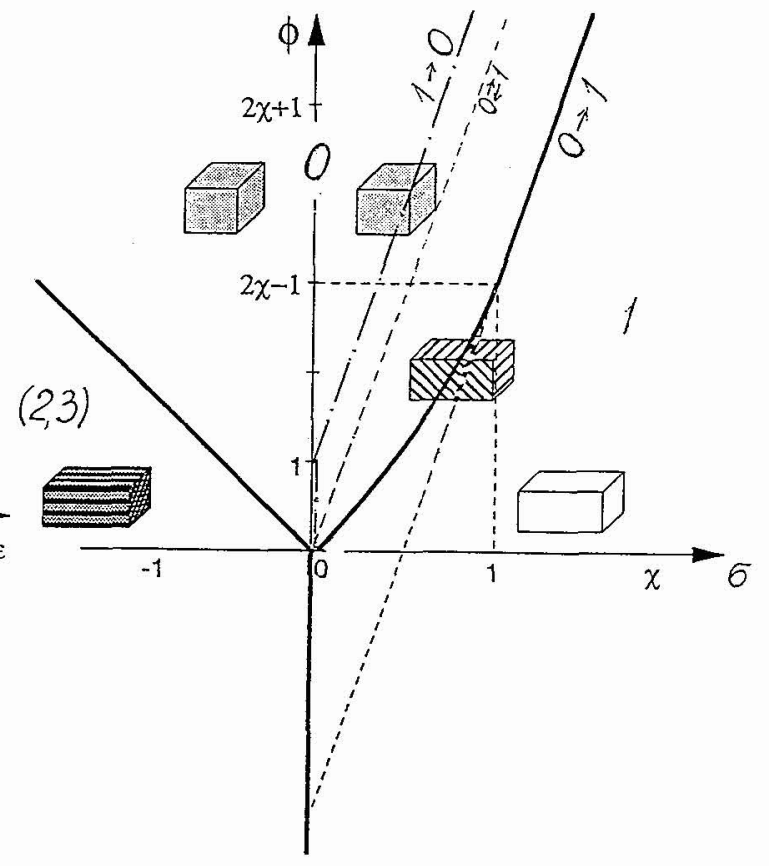


The combined line $\mathrm{g}^{*}(\sigma)$ (Eqs.(4.6),(4.7)) determines the unstable states which a metastable phase should overcome to be transformed into a stable one at fixed stress. The thermodynamic barrier $\Delta \mathrm{g}=\mathrm{g}^{*}-\mathrm{g}_{1}$ disappears at $\beta^{*}=1-\delta / \sqrt{ } 2 \gamma=0$ or $\sigma_{1}=\sqrt{ }(\chi-1)^{2}+\phi-(\chi-1)$ (compare with Eq.(3.11)). The cubic phase becomes unstable at $\sigma>\sigma_{1}$ (Fig.9b).

On the other side $\Delta \mathrm{g}=\mathrm{g}^{*}-\mathrm{g}_{0} \Rightarrow 0$ at $\beta^{*}=\delta+1 / 2=1$ or $\sigma=(\phi+1) / 2 \chi$ (compare with Eq. (3.18)). The tetragonal phase loses thermodynamical stability with respect to the transformation into the cubic one. The phase state between these end points of $\mathrm{g}^{*}$ depends on the direction of the transformation.

The dependence of strain on stress

$$
\varepsilon=-\mathrm{dg} / \mathrm{d} \sigma=\sqrt{ } 2 \gamma+(\chi-1)(1-\sigma / 2 \gamma)
$$

is a reciprocal function of $\sigma(\varepsilon)$, considered in part 3. Now a negative Young's modulus can be easily expressed as a function of $\sigma$ as follow:

$$
E_{\text {eff }}=\left(\frac{d \varepsilon}{d \sigma}\right)^{-1}=-\frac{1}{(\chi-1)} \frac{\left(\sqrt{2 \gamma}^{3}\right.}{4 \gamma+\sigma(\chi-1)}
$$

For example:

$$
E_{\text {eff }}\left(\sigma_{1}\right)=-\frac{1}{(\chi-1)} \frac{\sigma_{1}^{2}}{2 \sigma_{1}+(\chi-1)}
$$

(compare with Eq.(3.12)).

If $\phi>2 \chi-1 \sigma_{3}$ moves beyond the end point $\sigma_{1}$, this point transforms to $\sigma_{1}{ }^{\prime}=(\phi-1) / 2 \chi$ and unstable states consist of the single domain tetragonal phase. At $\phi \leq 1$ the section of $\mathrm{g}^{*}(\sigma)$ (Eq.(4.7)) corresponding to the single domain states disappears and $\sigma_{2} \rightarrow \sigma_{2}^{\prime}=0$. It means that the cubic phase is formed from the polytwin tetragonal phase.

Thus, a hysteresis loop should be observed for stress controlled deformation instead of a section of $\sigma-\varepsilon$ curve with negative slope for strain controlled deformation (Fig.10). The horizontal branches of the loop correspond to unstable states of the heterophase system. Their positions correspond to the maximum stress $\sigma_{1}$ on loading and the minimum stress $\sigma_{3}$ on unloading. $\sigma_{1}$ is the yield stress of the cubic phase when the first polytwin plates (if $\phi<2 \chi-1$ ) or single domain plates $(\phi>2 \chi-1$ ) are formed. The yield stress of the reverse transformation always corresponds to the formation of single domain plates. But if $\phi<1$, the cubic phase forms from the polytwin tetragonal one. In this case, the reverse stress is zero.

$$
\begin{aligned}
& \text { Inverting } \sigma_{1}(\phi) \text { and } \sigma_{3}(\phi)(\text { Eqs.(3.13),(3.19),(3.18)): } \\
& \qquad \begin{aligned}
\phi_{1}= & \sigma_{1}^{2}+2(\chi-1) \sigma \\
\phi_{1}^{\prime}= & 2 \chi \sigma-1 \\
\phi_{3}= & 2 \chi \sigma+1
\end{aligned}
\end{aligned}
$$

determines the temperatures of onsets of the transformations at fixed external stress $\sigma$ (Fig.11). The transformation of tetragonal phase begins at temperature, $\phi_{3}$, higher than the temperature of the phase equilibrium which is determined by the Clapeyron-Clausius equation $\mathrm{d} \Delta \mathrm{f}=\varepsilon_{0} \mathrm{~d} \sigma\left(\mathrm{qdT}=\mathrm{T}_{0} \varepsilon_{0} \mathrm{~d} \sigma\right.$ ) or $\phi_{0}=2 \chi \sigma[8]$. The shift of the temperature $(\Delta \phi=1)$ is determined by the elastic energy $\left(e=1 / 2 \mathrm{E}_{2} \varepsilon_{0}{ }^{2}\right)$ which is necessary for the formation of a single domain plate of the cubic phase. The linear $\phi-\sigma$ dependence $\left(\phi_{3}(\sigma)\right)$ corresponds to the approximation of equal elastic moduli of the phases. Under the same 
approximation, the $\phi_{1}{ }^{\prime}(\sigma)$ stress dependence of the temperature of the transformation of the high symmetry phase to the low symmetry one is considerably nonlinear. It is a result of the formation of a polytwin plate which has a variable domain fraction and, therefore, its self-strain is dependent on the external stress applied before the transformation. At a stress $\sigma_{\mathrm{c}}=1$, and higher, the first plates of the tetragonal phase are single domain ones and the $\phi_{1}^{\prime}(\sigma)$ dependence on $\sigma$ becomes linear as it is in the case of the reverse transformation. At $\sigma_{c}=1$ the curvature $\mathrm{d}^{2} \phi / \mathrm{d} \sigma^{2}$ has a jump which corresponds to an abrupt change of compliance: in comparison with a single domain phase a polytwin phase has additional component of compliance due to changing domain fraction. So the singular point $\left(\phi_{c}=2 \chi-1, \sigma_{c}=1\right)$ on $\phi(\sigma)$ - curve shows morphological transition of the product phase from polytwin state to single domain one. From thermodynamical point of view this point is similar to a critical point where the line of second-order transition (between single and polytwin products, $\sigma=\sigma_{c}$ ) meets the line of first-order transition between the high and low symmetry phases.

Comparison of the $\phi-\sigma$ diagram (Fig.11) and the $\phi-\varepsilon$ diagram (Fig.5) shows that the "quasi eutectic" line reduces to the triple point $(\sigma=0, \phi=0)$. The three lines of equilibria between three isolated phases meet each other at this point: the phases are cubic (0), tetragonal single domain (1) and tetragonal polytwin $(2,3)$. For coherent phases the line $0 / 1$ splits into two lines: $0 \rightarrow 1$ transformation and $1 \rightarrow 0$ transformation.

It is necessary to note that the peculiarities of stress controlled deformation can be observed much easier than those of strain controlled deformation, in particular during uniaxial deformation. The hysteretic $\sigma-\varepsilon$ curve corresponding to the loops considered above were observed during deformation of the shape memory alloys [9].

Another experimental evidence supporting the theory is the observation of changing polytwin structure and orientation of the first martensite plates during martensitic transformation under load. The results obtained for the Fe-Ni alloy are in the good quantitative agreement with the predictions of this theory [10].

\section{CONCLUSION}

The approach presented in this paper can be generalized leading to the theory of transformational elasticity. The elastic properties of polydomain crystals are strongly nonlinear and anisotropic. An addition, the polydomain crystals must manifest the strong nonlocal response to nonuniform deformation. Therefore, the strain gradient effects have to be included in the elastic theory [11]. It will then be possible to extend the elastic theory of polydomain crystals toward polycrystals with nonuniform microstructures. A combined effort of physicists, mechanicians and mathematicians is needed to make progress on these fascinating problems.

\section{Acknowledgments}

The papers where one can find some additional explanations or proofs are given in the short list of references below. I also want to acknowledge the many implicit benefits I received from sources not mentioned in this list. They are: the theory of spinodal decomposition of J. Cahn and the theory of coherent phases (J.Cahn and F.Larche); theory of homogenization and optimal composites (R. Kohn, G.Milton, A.Cherkaev, L. Gibiansky); the invariant plane theory (represented by D.Lieberman), the theory of compatible microstructures (R. James, J. Ball, K.Bhattacharya) and the studies by I.Muller. Of course many experimental studies on shape memory effects and thermoelastic martensitic transformation which have provided important stimuli. Among of them I like to name the pioneer work by G. Kurdumov and $\mathrm{L}$. Handros where thermoelastic martensitic transformation was predicted and observed first.

I am very grateful to Manfrend Wuttig for fruitful and kind discussions. The encouragement of R.Barsoum from the Office of Naval Research and the support of this office under contract N00014-931056 are appreciated as well. 


\section{References}

1. Roytburd A.L., Phase Transitions 45 (1993) 1-33

2. Roitburd A.L., "Martensitic transformation as typical phase transformation in solids.", Solid State Physics v.33, F. Ehrenreich, D. Seitz, D.Turnbull (Eds) (Academic Press, New York, 1978), pp.317390

3. Khachaturyan A.G., Theory of Structural Transformation in Solids (John Wiley and Sons, New York, 1983)

4. Kohn R and Grabovsky Y., private communication

5. Roitburd A.L.and Kosenko N.S., Phys. Stat. Sol. (A) 35 (1976) 735

6. Weschler M.S., Lieberman D.S. and Read T.A. Trans AIME 197 (1953) 1503

7. Roytburd A.L. and Slutsker J., J. Appl. Phys. 77 (1995) 27-45; Scripta Metal .Mater. 32 (1995) 761-766

8. Roitburd A.L., Soviet Physics-Solid State 25 (1983) 17; Soviet Physics Izvestia 47 (1983) 17

9. Muller I. and Xu H., Acta Metall 39 (1991) 263-270

10. Roitburd A.L. and Pankova M.N., Phys. Metall.Metall. 59 (1985) 131

11.Roytburd A.L., Wuttig M., Zhukovskiy I., Scripta Metal. Mater. 27 (1992) N10. 